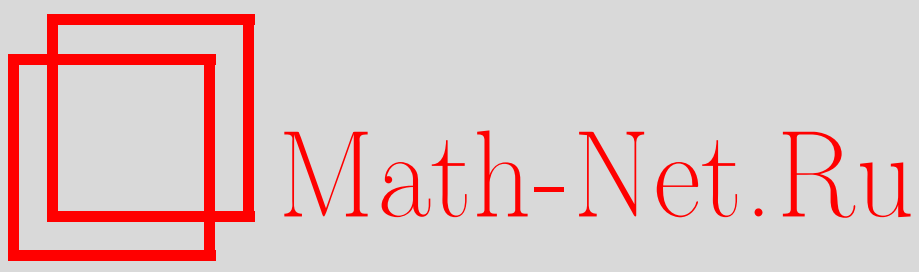

В. А. Скворцов, Аппроксимативная симметрическая вариация и $N$-свойство Лузина, Изв. РАН. Сер. матем., 1997, том 61, выпуск 4, 155-166

DOI: https://doi.org/10.4213/im140

Использование Общероссийского математического портала Math-Net.Ru подразумевает, что вы прочитали и согласны с пользовательским соглашением

http://www. mathnet.ru/rus/agreement

Параметры загрузки:

IP : 44.207 .124 .84

26 апреля 2023 г., 04:30:54 
УДК 517.518

\title{
В. А. Скворцов
}

\section{Аппроксимативная симметрическая вариация и $N$-свойство Лузина}

\begin{abstract}
Построен пример непрерывной функции, которая всюду имеет аппроксимативную симметрическую производную, но в то же время не обладает $N$-свойством Лузина. На том же примере доказано существование непрерывной функции, у которой аппроксимативная вариация на некотором множестве нулевой меры отлична от нуля, а аппроксимативная симметрическая вариация равна нулю на том же множестве.

Библиография: 7 наименований.
\end{abstract}

Известно (см. [1]), что если функция аппроксимативно дифференцируема на некотором множестве, то она является ACG-функцией на этом множестве и тем самым обладает на нем $N$-свойствами Лузина.

Здесь мы покажем, что этот результат не распространяется на симметрические аппроксимативные первообразные. Более того, в примере, который будет построен, аппроксимативная симметрическая производная построенной функции равна нулю на том множестве меры нуль, для которого нарушается $N$-свойство. Это позволяет на указанном примере провести также сравнение двух понятий вариации функции по множеству, определяемых с помощью двух соответствующих дифференциальных базисов: аппроксимативного и аппроксимативного симметрического.

Приведем необходимые определения (см. [2]-[4]).

Напомним, что по определению функция $F$ обладает на множестве $X$ $N$-свойством, если для любого подмножества $E \subset X$ меры нуль его образ $\mathscr{F}(E)$ также имеет меру нуль.

Дифференциальным базисом $B$ назовем некоторое семейство подмножеств декартова произведения $J \times \mathbb{R}$, где $J$ - множество всех невырожденных интервалов на действительной прямой $\mathbb{R}$. Базис $B$ будем предполагать фильтрующимся, т.е. обладающим свойством: для любых двух элементов $\beta_{1}, \beta_{2} \in B$ существует такой элемент $\beta$, что $\beta \subset \beta_{1} \cap \beta_{2}$ (предполагается также, что пустое множество не содержится в $B$ ). Для $E \subset \mathbb{R}$ введем обозначение

$$
\beta[E]=\{(I, x) \in \beta, \quad x \in E\} .
$$

Работа выполнена при поддержке РФФИ (проект № 94-01-00417).

$$
\text { (C) В. А. Скворцов } 1997
$$


Пусть $\Phi$ - функция интервала, заданная на всех интервалах $I$ таких, что $(I, x) \in \beta$ при некотором $x$ и некотором $\beta$. Определим верхнее и нижнее производные числа функчии $\Phi$ в точке $x$ относительно базиса $B$, положив

$$
\begin{aligned}
& \bar{D}_{B} \Phi(x)=\inf _{\beta \in B} \sup _{(I, x) \in \beta[\{x\}]} \frac{\Phi(I)}{|I|}, \\
& \underline{D}_{B} \Phi(x)=\sup _{\beta \in B} \inf _{(I, x) \in \beta[\{x\}]} \frac{\Phi(I)}{|I|} .
\end{aligned}
$$

Будем предполагать, что для любой точки $x \in \mathbb{R}$ и для любого $\beta \in B$ справедливо $\beta[\{x\}] \neq \varnothing$. Тогда всегда $\bar{D}_{B} \Phi(x) \geqslant \underline{D}_{B} \Phi(x)$. Если $\bar{D}_{B} \Phi(x)=\underline{D}_{B} \Phi(x)$, то это общее значение называется производной функиии $\Phi$ по базису $B$ в точке $x$ и обозначается $D_{B} \Phi(x)$.

Вариация функиии интервала $\Phi$ по множеству $E \subset \mathbb{R}$ относительно $\beta \in B$ определяется соотношением

$$
V(\Phi, E, \beta)=\sup \sum_{j} \Phi\left(I_{j}\right)
$$

где sup берется по всевозможным конечным наборам неперекрывающихся интервалов $I_{j}$ таких, что $\left(I_{j}, x_{j}\right) \in \beta[E]$.

Вариачия функции $\Phi$ по множеству $E \subset \mathbb{R}$ относительно базиса $B$ определяется соотношением

$$
V(\Phi, E, B)=\inf _{\beta \in B} V(\Phi, E, \beta) .
$$

Для функции точки $F$ понятия производных чисел и вариации определяются через соответствующие понятия для функции интервала, заданной для интервала $I=[a, b]$ равенством

$$
\Phi(I)=F(b)-F(a) .
$$

Здесь мы будем рассматривать определенные вьше понятия применительно к двум дифференциальным базисам: аппроксимативному и симметрическому аппроксимативному. Для описания этих базисов введем вспомогательное понятие плотностной шкалы.

Плотностной икалой $\Delta$ на измеримом множестве $E \subset \mathbb{R}$ назовем измеримое подмножество плоскости $\mathbb{R}^{2}$ такое, что для каждого $x \in E$ множество $\Delta_{x}=$ $\{h, h>0,(x, h) \in \Delta\}$ имеет внутреннюю правую плотность 1 в точке 0 (предположение измеримости $\Delta$ используется лишь при определении аппроксимативного симметрического интеграла, оно не понадобится нам в данной статье).

Для фиксированной плотностной шкалы $\Delta$ на отрезке $[a, b]$ определим множество $\beta_{\Delta}$ всевозможных пар $([x, x \pm h], x)$, где $(x, h) \in \Delta$. Эти множества $\beta_{\Delta}$ и будут при всевозможных $\Delta$ выступать в качестве элементов аппроксимативного базиса $B_{\text {ар. }}$ Аналогичным образом аппроксимативный симметрический базис $B_{\text {ap.s }}$ определяется как набор элементов $\beta_{\Delta}^{s}$ для всевозможных шкал $\Delta$, каждый из которых представляет собой множество всех пар $([x-h, x+h], x)$, где $(x, h) \in \Delta$ для фиксированной шкалы $\Delta$. 
Определение (1), примененное к элементу $\beta_{\Delta}$ аппроксимативного базиса $B_{\mathrm{ap}}$ и к функции множества $\Phi$, заданной равенством (3) по функции точки $F$, определенной на некотором интервале, сводится к выражению

$$
V\left(F, E, \beta_{\Delta}\right)=\sup \sum_{j}\left|F\left(x_{j} \pm h_{j}\right)-F\left(x_{j}\right)\right|
$$

где sup берется по множеству всех конечных наборов неперекрываюшихся интервалов вида $\left[x_{j}-h_{j}, x_{j}\right]$ или $\left[x_{j}, x_{j}+h_{j}\right]$ при $x_{j} \in E$ и $\left(x_{j}, h_{j}\right) \in \Delta$. Тогда в соответствии с определением (2) аппроксимативная вариация функции $F$ на множестве $E$ определяется как

$$
V_{\mathrm{ap}}(F, E)=\inf \left(F, E, \beta_{\Delta}\right),
$$

где inf берется по множеству всех элементов базиса $B_{\text {ap }}$, т.е. по множеству всех плотностных шкал.

Аналогичным образом конкретизируется определение аппроксимативной симметрической вариации. А именно, сначала полагаем

$$
V\left(F, E, \beta_{\Delta}^{s}\right)=\sup \sum_{j}\left|F\left(x_{j}+h_{j}\right)-F\left(x_{j}-h_{j}\right)\right|,
$$

где sup берется по множеству всех конечных наборов неперекрываюшихся интервалов вида $\left[x_{j}-h_{j}, x_{j}+h_{j}\right]$ при $x_{j} \in E$ и $\left(x_{j}, h_{j}\right) \in \Delta$, а затем определяем $a n-$ проксимативную симметрическую вариачию как

$$
V_{\text {ap.s }}(F, E)=\inf _{\Delta} V\left(F, E, \beta_{\Delta}^{s}\right) .
$$

Определение производных чисел и производной, примененное к базисам $B_{\text {ap }}$ и $B_{\text {ap.s. }}$, дает понятие аппроксимативных и соответственно аппроксимативных симметрических производных чисел и производной $D_{\mathrm{ap.s}} F(x)$.

Непосредственно из определений производной и вариации легко выводится следующее утверждение.

ЛЕмма 1. Если на множестве Е аппроксимативная или аппроксимативная симметрическая производная функции F равна нулю, то соответствующая вариащия функции $F$ на множестве $E$ также равна нулю.

Приступим теперь к описанию конструкции примера, из которого будут получены основные результаты данной статьи.

Прежде всего построим на $[0,1]$ совершенное симметричное множество канторовского типа с переменньм отношением длины удаляемого интервала к длине отрезка, из которого он удаляется. Все интервалы и отрезки в нижеследующем построении имеют два индекса, где верхний означает ранг (шаг построения), а нижний - порядковый номер слева направо при фиксированном верхнем индексе. Поскольку все интервалы одного и того же ранга будут иметь одинаковую длину, то мы можем условиться обозначать длину интервала той же буквой, что и сам интервал, но без нижнего индекса. Так, $u^{(n)}$ будет обозначать длину любого из интервалов $u_{j}^{(n)}$. 
Построение проведем по индукции. Пусть $s_{0}^{(1)}=[0,1]$. Удалим из $s_{0}^{(1)}$ концентрический с ним интервал $u_{0}^{(1)}$ так, чтобы длина каждого из двух остающихся отрезков второго ранга, $s_{0}^{(2)}$ и $s_{1}^{(2)}$, была равна $s^{(2)}=2^{-1} \cdot 2^{-2}=2^{-1} \cdot 2^{-2} s^{(1)}$. Пусть после $n-1$ шагов остались отрезки $s_{j}^{(n)}, j=0,1, \ldots, 2^{n-1}-1$, ранга $n$ длины $s^{(n)}$. Удалим из каждого из них конщентрический с ним интервал $u_{j}^{(n)}$ длины

$$
u^{(n)}=\left(1-(n+1)^{-2}\right) s^{(n)}
$$

после чего останется $2^{n}$ отрезков $s_{j}^{(n+1)}, j=0,1, \ldots, 2^{n}-1$, ранга $n+1$ длины

$$
s^{(n+1)}=2^{-1}(n+1)^{-2} s^{(n)}
$$

причем

$$
s_{2 j}^{(n+1)} \cup u_{j}^{(n)} \cup s_{2 j+1}^{(n+1)}=s_{j}^{(n)}
$$

Положим

$$
S_{n}=\bigcup_{j=0}^{2^{n-1}-1} s_{j}^{(n)}, \quad S=\bigcap_{n=1}^{\infty} S_{n} .
$$

Легко проверить, что

$$
s^{(n+1)} \leqslant 2^{-1} \cdot 2^{-2} s^{(n)} \leqslant 2^{-n} \cdot 2^{-2 n} s^{(1)}=2^{-n} \cdot 2^{-2 n},
$$

откуда $\left|S_{n}\right| \leqslant 2^{-2(n-1)}$, и поэтому

$$
|S|=0 \text {. }
$$

Добавим к семейству смежных интервалов $\left\{u_{j}^{(n)}\right\}$ два несобственных интервала, $u_{-1}^{(0)}=(-1,0)$ и $u_{0}^{(0)}=(1,2)$, так что все дальнейшее построение осуществим на интервале $(-1,2)$.

Заметим, что к каждому отрезку $s_{j}^{(n)}, n=1,2, \ldots ; j=0,1, \ldots, 2^{n-1}-1$, примыкают слева и справа смежные интервалы ранга, не превосходящего $n-1$, причем ранг одного из них (при $n \geqslant 2$ ) в точности равен $n-1$. На интервале $u_{l}^{(k)}$, расположенном слева от $s_{j}^{(n)}$, отложим интервал $t_{2 j}^{(n)}$ так, чтобы его правый конец совпал с левым конщом отрезка $s_{j}^{(n)}$, а на интервале $u_{p}^{(m)}$, примыкаюшем к $s_{j}^{(n)}$ справа, отложим интервал $t_{2 j+1}^{(n)}$, левый конец которого совпадает с правым концом отрезка $s_{j}^{(n)}$. При этом длина откладываемых интервалов удовлетворяет соотношению

$$
t^{(n+1)}=4^{-1} \cdot n^{-1} u^{(n)}=4^{-1} n^{-1}\left(1-(n+1)^{-2}\right) s^{(n)}
$$

(второе равенство следует из (8)).

Отметим, что

$$
t^{(n+1)}=o\left(u^{(n)}\right) \quad \text { при } \quad n \rightarrow \infty .
$$


Поскольку каждый интервал $u_{j}^{(n)}$ по построению заключен между $s_{2 j}^{(n+1)}$ и $s_{2 j+1}^{(n+1)}$ (см. $(10))$, на нем будут отложены интервалы $t_{4 j+1}^{(n+1)}$ и $t_{4 j+2}^{(n+1)}$. Между ними будет заключен отрезок, конщентрический с $u_{j}^{(n)}$, который мы обозначим $v_{j}^{(n)}$. Итак,

$$
u_{j}^{(n)}=t_{4 j+1}^{(n+1)} \cup v_{j}^{(n)} \cup t_{4 j+2}^{(n+1)}
$$

В силу (12)

$$
v^{(n)}=\left(1-2^{-1} n^{-1}\right) u^{(n)}>0 \quad \text { при всех } \quad n=1,2, \ldots
$$

Но к интервалу $u_{j}^{(n)}$ примыкают также отрезки $s_{k}^{(m)}$ ранга $m$ при всех $m \geqslant n+1$. Поэтому в результате построения на $u_{j}^{(n)}$ будут отложены интервалы длины $t^{(m)}$, $m \geqslant n+1$, образуюшие две последовательности вложенных интервалов:

$$
\left\{t_{j_{m}}^{(m)}\right\}_{m} \quad \text { и } \quad\left\{t_{j_{m}+1}^{(m)}\right\}_{m}
$$

так что интервалы первой последовательности имеют с $u_{j}^{(n)}$ левый общий конец, а интервалы второй последовательности - правый общий конец. Тем самым интервал $u_{j}^{(n)}, n \geqslant 1$, может быть представлен в виде следующего объединения непересекающихся промежутков:

$$
u_{j}^{(n)}=v_{j}^{(n)} \cup\left(\bigcup_{m=n+1}^{\infty}\left(t_{j_{m}}^{(m)} \backslash t_{j_{m+1}}^{(m+1)}\right)\right) \cup\left(\bigcup_{m=n+1}^{\infty}\left(t_{j_{m}+1}^{(m)} \backslash t_{j_{m+1}+1}^{(m+1)}\right)\right) .
$$

На интервале $u_{-1}^{(0)}$ отложены интервалы $t_{0}^{(n)}=\left(-t^{(n)}, 0\right), n=1,2, \ldots$, а на $u_{0}^{(0)}$ интервалы $t_{2^{n+1}-1}^{(n)}=\left(1,1+t^{(n)}\right), n=1,2, \ldots$, так что

$$
\begin{aligned}
& u_{-1}^{(0)}=(-1,0)=\left(-1,-t^{(1)}\right] \cup\left(\bigcup_{n=1}^{\infty} t_{0}^{(n)}\right), \\
& u_{0}^{(0)}=(1,2)=\left(\bigcup_{n=1}^{\infty} t_{2^{n+1}-1}^{(n)}\right) \cup\left[1+t^{(1)}, 2\right) .
\end{aligned}
$$

Отметим следуюшие соотношения. Из (9) и (12) следует равенство

$$
s^{(n)}=2^{-1} n^{-2} s^{(n-1)}=2(n+1)^{-1} t^{(n)},
$$

так что

$$
\begin{array}{cl}
s^{(n)}=o\left(t^{(n)}\right) & \text { при } \quad n \rightarrow \infty, \\
s^{(n)} \leqslant 4^{-1} t^{(n)} & \text { при всех } \quad n \geqslant 3 .
\end{array}
$$


Из (12) следует, что

$$
t^{(n+1)} \leqslant 4^{-1} s^{(n)} \quad \text { при всех } \quad n \geqslant 1 .
$$

Отсюда при $n \geqslant 3$

$$
s^{(n)} \leqslant 2^{-4} s^{(n-1)}, \quad t^{(n+1)} \leqslant 2^{-4} t^{(n)},
$$

и поэтому

$$
\sum_{k=n}^{\infty} t^{(k)} \leqslant 2 t^{(n)}, \quad \sum_{k=n}^{\infty} s^{(k)} \leqslant 2 s^{(n)} .
$$

Определим на множестве $S$ сингулярную меру $\mu$ так, чтобы для любого $n \geqslant 1$

$$
\mu\left(S \cap s_{j}^{(n)}\right)=2^{1-n} .
$$

В частности,

$$
\mu(S)=\mu\left(S \cap s_{0}^{(1)}\right)=1 .
$$

Зададим на $(-1,2)$ сингулярную непрерывную функцию

$$
R(x)=\mu(S \cap(-1, x]) .
$$

Приступим теперь к построению основной функции $F$.

Положим $F(x)=R(x)$, если $x \in S$, и сразу отметим, что отсюда, из (11) и (24) следует

Свойство 1. Функция $F$ отображает множество $S$ на отрезок $[0,1]$ и тем самым не обладает $N$-свойством.

Пусть также

$$
\begin{gathered}
F(x)=R(x) \text { при } x \in v_{j}^{(n)}, \quad n=1,2, \ldots, \\
j=0,1, \ldots, 2^{n-1}-1 \text { и при } x \in\left(-1,-t^{(1)}\right] \cup\left[1+t^{(1)}, 2\right) .
\end{gathered}
$$

В остальных точках каждого интервала $u_{j}^{(n)}$ определим функцию $F$ с учетом представлений (16) и $(17)$ и в зависимости от уже заданных значений $F$ на отрезках $v_{k}^{(m)}$ в соседних смежных интервалах. Эти постоянные в силу (26) и $(25)$ значения $F$ в точках отрезка $v_{k}^{(m)}$ будет удобно обозначить $F\left(v_{k}^{(m)}\right)$.

Легко проверить, что индексы в последовательностях (16) удовлетворяют соотношениям $j_{m+1}=2 j_{m}+1, j_{m+1}+1=2\left(j_{m}+1\right)$. В частности, отсюда видно, что если $t_{2 j}^{(m)}$ и $t_{2 j+1}^{(m)}$ примыкают к отрезку $s_{j}^{(m)}$ соответственно слева и справа, то

$$
t_{2 j}^{(m)} \supset t_{4 j}^{(m+1)}, \quad t_{2 j+1}^{(m)} \supset t_{4 j+3}^{(m+1)}
$$

Заметив это и вспомнив, что $v_{j}^{(m)} \subset u_{j}^{(m)} \subset s_{j}^{(m)}$, положим

$$
F(x)=F\left(v_{j}^{(m)}\right)=R\left(v_{j}^{(m)}\right), \quad x \in\left(t_{2 j}^{(m)} \backslash t_{4 j}^{(m+1)}\right) \cup\left(t_{2 j+1}^{(m)} \backslash t_{4 j+3}^{(m+1)}\right) .
$$


Для дальнейшего отметим, что если $t_{l}^{(m+1)}$ и $t_{i}^{(m+2)} \subset t_{l}^{(m+1)}$ - интервалы, лежащие на некотором смежном интервале $u_{q}^{(m)}$, а к ним примыкает отрезок $s_{p}^{(m+1)}$ с лежашими на нем интервалом $u_{p}^{(m+1)}$, отрезком $v_{p}^{(m+1)} \subset u_{p}^{(m+1)}$ и отрезком $s_{k}^{(m+2)}$, отделяющим $u_{q}^{(m)}$ от $u_{p}^{(m+1)}$, то (см. $(23)$ и $\left.(25)\right)$

$$
\left|R\left(v_{p}^{(m+1)}\right)-R\left(v_{q}^{(m)}\right)\right|=\mu\left(S \cap s_{k}^{(m+2)}\right)=2^{-m-1},
$$

и поэтому, с учетом (26) и (27),

$$
\left|F\left(t_{l}^{(m+1)} \backslash t_{i}^{(m+2)}\right)-F\left(v_{q}^{(m)}\right)\right|=2^{-m-1}, \quad t_{l}^{(m+1)} \cup v_{q}^{(m)} \subset u_{q}^{(m)} .
$$

Итак, функция $F$ определена на всем интервале $(-1,2)$, но, будучи кусочно постоянной на каждом смежном интервале $u_{j}^{(n)}$, она имеет изолированные разрывы в концах интервалов $t_{k}^{(m)}$, лежаших в $u_{j}^{(n)}$. Окружим каждую точку изолированного разрыва столь малой окрестностью, чтобы сумма всех этих окрестностей, взятая по всем смежным интервалам, образовывала множество $P$, имеющее нулевую плотность в каждой точке $x \in S$ и не влияюшее тем самым на значения аппроксимативной производной в точках $S$. Изменяя далее значения функции $F$ в указанных окрестностях, сгладим $F$ так, чтобы она, принимая внутри каждой из этих окрестностей значения, промежуточные между значениями в концах окрестности, была дифференцируемой всюду внутри каждого смежного интервала $u_{j}^{(n)}$. Договоримся полученную в результате исправления функцию по-прежнему обозначать $F$.

Заметим, что в силу (16) и (27) и ввиду очевидной непрерывности функции $R$ функция $F$ непрерывна в концевых точках интервалов $u_{j}^{(n)}$ изнутри этих интервалов. Кроме того, из определения функции $F$ видно, что ее значения на интервале $u_{j}^{(n)}$ определяются значениями функции $R$ на примыкающих к $u_{j}^{(n)}$ отрезках $s_{k}^{(m)}$ ранга $m$ при $m>n$. Поскольку все эти отрезки принадлежат отрезку $s_{j}^{(n)}$, то колебание функции $F$ на $u_{j}^{(n)}$ не превосходит колебания функции $R$ на $s_{j}^{(n)}$, т.е. в силу $(23)$ и $(25)$ не превосходит $2^{1-n}$ и, значит, стремится к нулю при $n \rightarrow \infty$. Тем самым можно заключить, что функция $F$ непрерывна на $(-1,2)$.

Покажем теперь, что

$$
D_{\text {ap.s }} F(x)=0 \quad \text { при } \quad x \in S .
$$

Для этого достаточно показать, что для каждой точки $x \in S$ плотность множества $E(x)=\{y: y>0, F(x-y) \neq F(x+y)\}$ равна нулю в нуле.

Рассмотрим множество $E_{n}^{(1)}(x)=E(x) \cap\left(t^{(n)}, u^{(n-1)}-t^{(n)}\right]$. Возьмем отрезок $s_{j}^{(n)}$, для которого $x \in s_{j}^{(n)}$. Один из примыкающих к $s_{j}^{(n)}$ смежных интервалов имеет ранг $n-1$. Пусть для определенности он примыкает к $s_{j}^{(n)}$ справа. Обозначим его $u_{k}^{(n-1)}$. Смежный интервал, примыкающий к $s_{j}^{(n)}$ слева, имеет в этом случае ранг $m<n-1$. На этих интервалах лежат, примыкая к $s_{j}^{(n)}$ слева и 
справа, соответственно интервалы $t_{2 j}^{(n)}$ и $t_{2 j+1}^{(n)}$, а на левом смежном интервале находится также интервал $t_{2 k}^{(n-1)} \supset t_{2 j}^{(n)}$. В силу того, что при достаточно большом $n$ справедливо неравенство $u^{(n-1)}-t^{(n)}=t^{(n)}+v^{(n-1)}<s^{(n-1)}<t^{(n-1)}($ см. $(20))$, окрестность радиуса $u^{(n-1)}-t^{(n)}$ рассматриваемой точки $x \in S$ удовлетворяет соотношению

$\left(x-u^{(n-1)}+t^{(n)}, x+u^{(n-1)}-t^{(n)}\right) \subset\left(t_{2 k}^{(n-1)} \backslash t_{2 j}^{(n)}\right) \cup t_{2 j}^{(n)} \cup s_{j}^{(n)} \cup t_{2 j+1}^{(n)} \cup v_{k}^{(n-1)}$.

В силу (27) при $m=n-1$, если $x+y \in v_{k}^{(n-1)}$ и $x-y \in t_{2 k}^{(n-1)} \backslash t_{2 j}^{(n)}$, то

$$
F(x+y)=F(x-y)=F\left(v_{k}^{(n-1)}\right)
$$

Таким образом, в рассматриваемом случае в $E_{n}^{(1)}(x)$ могут входить лишь точки $y$, при которых хотя бы одна из точек $x+y$ и $x-y$ попадает в множество $t_{2 j}^{(n)} \cup s_{j}^{(n)} \cup t_{2 j+1}^{(n)} \cup P$. Но поскольку $x \in s_{j}^{(n)}$, то это может случиться лишш тогда, когда $y<t^{(n)}+s^{(n)}$. Поэтому $E_{n}^{(1)}(x) \subset\left(t^{(n)}, t^{(n)}+s^{(n)}\right)$ и, следовательно,

$$
\left|E_{n}^{(1)}(x)\right| \leqslant s^{(n)}
$$

Далее рассмотрим множество $E_{n}^{(2)}(x)=E(x) \cap\left(u^{(n-1)}-t^{(n)}, t^{(n-1)}\right]$. Пусть $x \in s_{j}^{(n-1)}$. Окрестность точки $x$ радиуса $t^{(n-1)}$ удовлетворяет соотношению

$$
\left(x-t^{(n-1)}, x+t^{(n-1)}\right) \subset\left(t_{2 j}^{(n-1)} \backslash t_{4 j}^{(n)}\right) \cup t_{4 j}^{(n)} \cup s_{j}^{(n-1)} \cup t_{4 j+3}^{(n)} \cup\left(t_{2 j+1}^{(n-1)} \backslash t_{4 j+3}^{(n)}\right) .
$$

Вновь воспользуемся соотношением (27), заменяя в нем $m$ на $n-1$. Имеем

$$
F(x+y)=F(x-y)=F\left(v_{j}^{(n-1)}\right), \quad x-y \in t_{2 j}^{(n-1)} \backslash t_{4 j}^{(n)}, \quad x+y \in t_{2 j+1}^{(n-1)} \backslash t_{4 j+3}^{(n)} .
$$

Хотя бы одно из последних соотношений может не выполняться лишь в случае, когда $h<s^{(n-1)}+t^{(n)}$. Поэтому

$$
E_{n}^{(2)}(x) \subset\left(u^{(n-1)}-t^{(n)}, s^{(n-1)}+t^{(n)}\right),
$$

и, значит, с учетом (10) и (18) при достаточно большом $n$,

$$
\left|E_{n}^{(2)}(x)\right| \leqslant s^{(n-1)}-u^{(n-1)}+2 t^{(n)}<4 t^{(n)}
$$

В силу (30), (31), (20) и (22) получаем, что при

$$
u^{(n-1)}-t^{(n)}<h \leqslant t^{(n-1)}
$$


и достаточно большом $n$ справедлива оценка

$$
\begin{aligned}
|E(x) \cap(0, h)| & \leqslant \sum_{k=n}^{\infty}\left|E_{k}^{(2)}(x)\right|+\sum_{k=n}^{\infty}\left|E_{k}^{(1)}(x)\right| \\
& \leqslant 4 \sum_{k=n}^{\infty} t^{(k)}+\sum_{k=n}^{\infty} s^{(k)} \leqslant 5 \sum_{k=n}^{\infty} t^{(k)} \leqslant 10 t^{(n)} .
\end{aligned}
$$

Поэтому, с учетом (13),

$$
\frac{|E(x) \cap(0, h)|}{h} \leqslant \frac{10 t^{(n)}}{u^{(n-1)}-t^{(n)}}=o(1), \quad n \rightarrow \infty
$$

при $h$, удовлетворяющих (32).

При

$$
t^{(n)}<h \leqslant u^{(n-1)}-t^{(n)}
$$

в силу $(30),(31),(21)$ и $(22)$ имеем

$$
\begin{aligned}
|E(x) \cap(0, h)| & \leqslant \sum_{k=n}^{\infty}\left|E_{k}^{(1)}(x)\right|+\sum_{k=n+1}^{\infty}\left|E_{k}^{(2)}(x)\right| \\
& \leqslant \sum_{k=n}^{\infty} s^{(k)}+4 \sum_{k=n+1}^{\infty} t^{(k)} \leqslant 2 \sum_{k=n}^{\infty} s^{(k)} \leqslant 4 s^{(n)}
\end{aligned}
$$

и поэтому в силу (19)

$$
\frac{|E(x) \cap(0, h)|}{h} \leqslant \frac{4 s^{(n)}}{t^{(n)}}=o(1), \quad n \rightarrow \infty,
$$

при $h$, удовлетворяющих (34).

Из (33) и (35) следует, что множество $E(x)$ имеет плотность нуль в нуле для каждой точки $x \in S$, откуда, как уже отмечалось, следует (29).

Итак, с учетом свойства 1 , мы доказали справедливость следующего утверждения.

ТЕОРема 1. Существует непрерывная функиия $F$, для которой аппроксимативная симметрическая производная $D_{\text {ар.s }} F(x)$ всюду конечна $и$ $D_{\text {ap.s }} F(x)=0$ на совершенном множестве $S$ нулевой меры. При этом функиия $F$ отображает множество $S$ в отрезок $[0,1]$ и тем самым не обладает $N$-свойством.

Рассмотрим теперь вариации $V_{\text {ap }}(F, S)$ и $V_{\text {ap.s }}(F, S)$ построенной функции $F$ (см. $(4)-(7))$. 
В силу леммы $1 V_{\text {ap.s }}(F, S)=0$. Покажем, что вариация $V_{\text {ap }}(F, S)$ отлична от нуля. Для этого оценим снизу вариацию $V\left(F, S, \beta_{\Delta}\right)$ для произвольной шкалы $\Delta$. Для каждого $x \in S$ найдем $\delta(x)>0$ так, чтобы

$$
\left|\Delta_{x} \cap(0, h)\right|>\frac{3}{4} h \quad \text { при } \quad 0<h<\delta(x) .
$$

Пусть $\left\{I_{k}\right\}_{k}=\left\{\left[x_{i}-h_{i}, x_{i}\right],\left[x_{j}, x_{j}+h_{j}\right]\right\}_{i, j}-$ конечный набор непересекаюшихся отрезков таких, что $x_{i}, x_{j} \in S, 0<h_{i}<\delta\left(x_{i}\right)$ и $0<h_{j}<\delta\left(x_{j}\right)$, причем

$$
S \subset \bigcup_{k} I_{k}
$$

и $x_{k}-$ конец отрезка $I_{k}$, для которого

$$
\left|I_{k}\right|<\delta\left(x_{k}\right)
$$

Существование такого покрытия доказывается стандартным способом. А именно, доопределим функцию $\delta(x)$ в точках дополнения к $S$ на $[0,1]$, положив ее в каждой такой точке $x$ равной расстоянию от $x$ до $S$. Затем возьмем покрытие всего отрезка непересекаюшимися отрезками вида

$$
[x-h, x], \quad[x, x+h] \quad \text { при } \quad 0<h<\delta(x)
$$

(существование такого покрытия установлено, например, в [5]). При этом в силу определения $\delta(x)$ точки множества $S$ будут покрыты лишь такими интервалами вида (39), для которых $x \in S$.

Для фиксированного интервала $I_{k}$ из покрытия (37) возьмем минимальное $n$, при котором некоторый отрезок $s_{j_{k}}^{(n)}$ целиком содержится в $I_{k}$ (мы, очевидно, можем исключить из покрытия отрезки, имеющие в пересечении с $S$ лишь одну точку). Поскольку в этом случае $I_{k}$ может иметь непустое пересечение лишь не более чем с двумя отрезками ранга $n-1$, то (см. (23))

$$
\mu\left(S \cap I_{k}\right) \leqslant 2 \mu\left(S \cap s_{j}^{(n-1)}\right)=2^{3-n} .
$$

Пусть $x_{k}$ - конец отрезка $I_{k}$, для которого выполняется (38), - лежит на отрезке $s_{p}^{(n+1)}$, и пусть $u_{q}^{(m)}$ - ближайший к $x_{k}$ интервал ранга $m \leqslant n$, целиком содержашийся в $I_{k}$. Очевидно, $u_{q}^{(m)}$ примыкает к $s_{p}^{(n+1)}$, и поэтому расстояние от $x_{k}$ до $u_{q}^{(m)}$ не превосходит $s^{(n+1)}$. К отрезку $s_{p}^{(n+1)}$ примыкают лежашие на $u_{q}^{(m)}$ и тем самьм целиком расположенные внутри $I_{k}$ интервалы $t_{l}^{(m+1)}$ и $t_{i}^{(m+2)} \subset$ $t_{l}^{(m+1)}$. Поэтому при $m \geqslant 3$ (см. (20) и (38))

$$
s^{(n+1)}+t^{(m+1)} \leqslant 2 t^{(m+1)}<u^{(m)}<\delta\left(x_{k}\right),
$$


и к $x_{k}$ применимо $(36)$ с $h=s^{(n+1)}+t^{(m+1)}$ и $h=u^{(m)}$. В то же время в силу (20), (21) и (14)

$$
t^{(m+1)}-t^{(m+2)} \geqslant \frac{1}{2}\left(s^{(n+1)}+t^{(m+1)}\right) \quad \text { и } \quad v^{(m)} \geqslant \frac{1}{2} u^{(m)} .
$$

Таким образом,

$$
\begin{aligned}
\left|\Delta_{x_{k}} \cap\left\{\left|y-x_{k}\right|: y \in t_{l}^{(m+1)} \backslash t_{i}^{(m+2)}\right\}\right| & \geqslant \frac{1}{4}\left(s^{(n+1)}+t^{(m+1)}\right), \\
\left|\Delta_{x_{k}} \cap\left\{\left|y-x_{k}\right|: y \in v_{q}^{(m)}\right\}\right| & \geqslant \frac{1}{4} u^{(m)},
\end{aligned}
$$

откуда следует, что найдется пара точек $y_{k}^{\prime} \in t_{l}^{(m+1)} \backslash t_{i}^{(m+2)}$ и $y_{k}^{\prime \prime} \in v_{q}^{(m)}$ таких, что для них числа $\left|y_{k}^{\prime}-x_{k}\right|$ и $\left|y_{k}^{\prime \prime}-x_{k}\right|$ принадлежат $\Delta_{x_{k}}$. Ввиду (28) справедливо соотношение

$$
\left|F\left(y_{k}^{\prime \prime}\right)-F\left(y_{k}^{\prime}\right)\right|=2^{-m-1} \geqslant 2^{-n-1} .
$$

Тогда одна из разностей $\left|F\left(y_{k}^{\prime}\right)-F\left(x_{k}\right)\right|$ и $\left|F\left(y_{k}^{\prime \prime}\right)-F\left(x_{k}\right)\right|$ должна быть не меньше $2^{-n-2}$. Обозначим через $y_{k}$ ту из точек $y_{k}^{\prime}$ и $y_{k}^{\prime \prime}$, для которой это неравенство реализуется. Тогда с учетом (40) справедливо неравенство

$$
\left|F\left(y_{k}\right)-F\left(x_{k}\right)\right| \geqslant 2^{-n-2} \geqslant 2^{-5} \mu\left(S \cap I_{k}\right),
$$

причем $\left|y_{k}-x_{k}\right| \in \Delta_{x_{k}}$. Если $I_{k}^{\prime}$ - интервал с концами $x_{k}$ и $y_{k}$, то $\left(I_{k}^{\prime}, x_{k}\right) \in \beta_{\Delta}$, и в соответствии с определением (4) и с учетом $(24),(37)$ и (41) имеем

$$
V\left(F, S, \beta_{\Delta}\right) \geqslant 2^{-5}
$$

откуда в силу произвольности $\Delta$ получаем, что $V_{\text {ap }}(F, S) \geqslant 2^{-5}$.

Итак, доказана

Tеорема 2. Существует непрерывная функиия, для которой аппроксимативная вариация на некотором множестве меры нуль отлична от нуля, а аппроксимативная симметрическая вариачия равна нулю на том же множестве.

Отметим к тому же, что в силу дифференцируемости построенной выше функции $F$ всюду вне множества $S$ мы можем утверждать, что вариация $V_{\text {ap.s }}(F, S)$ как функиия множества б-конечна на всем интервале $(-1,2)$, абсолютно непрерывна относительно меры Лебега, но при этом, не обладая $N$-свойством, не является $A C G$-функиией на $(-1,2)$ (см. по этому поводу проблему 29 из [4, c. 423], относящуюся к симметрической вариации).

В заключение воспользуемся построенной функцией $F$ для сравнения аппроксимативного симметрического интеграла с интегралом Данжуа-Хинчина.

Поскольку производная $D_{\text {ap.s }} F(x)=f(x)$ сушествует и конечна всюду на $[0,1]$, то $F(x)$, являясь точной аппроксимативной симметрической первообразной функции $f$, представляет собой неопределенньй интеграл функции $f$ как в смысле аппроксимативного симметрического интеграла Перрона (ASP-интеграла), так и в 
смысле аппроксимативного симметрического интеграла Хенстока (ASH-интеграла) (определения этих интегралов см. в [4]).

Рассмотрим теперь функцию $\Phi(x)=F(x)-R(x)$. Поскольку $R^{\prime}(x)=0$ всюду вне $S$, то

$$
\Phi^{\prime}(x)=F^{\prime}(x)=f(x) \quad \text { п.в. на }[0,1] .
$$

По построению $F$, а значит, и $\Phi$ являются ACG-функциями на каждом смежном интервале $u_{j}^{(n)}$. Кроме того, $\Phi(x)=0$ при $x \in S$, и, значит, $\Phi$ абсолютно непрерывна на $S$. Тем самым $\Phi$ является ACG-функцией на $[0,1]$. Вместе с (42) это показывает, что $\Phi$ является неопределенным интегралом функции $f$ на $[0,1]$ в смысле широкого интеграла Данжуа (см. [1]) и интеграла Данжуа-Хинчина (см. [5]), являющегося промежуточным между широким и узким интегралами Данжуа.

Таким образом, доказано следуюшее утверждение.

ТеОРема 3. АSP-интеграл (и АSH-интеграл) и интеграл Данжуа-Хинчина (DX-интеграл) могут противоречить друг другу на классе точных аппроксимативных симметрических производных, т.е. существует всюдуконечная интегрируемая в смысле $D X$-интеграла на $[0,1]$ функиия $f$, обладающая аппроксимативной симметрической первообразной $F$ с $D_{\text {ap.s }} F(x)=f(x)$ всюду на $[0,1]$, но для которой

$$
F(1)-F(0)=(A S P) \int_{0}^{1} f \neq(D X) \int_{0}^{1} f
$$

Заметим, что последнюю теорему другим способом можно также получить из результатов В.А. Скляренко (см. [6], а также [4, с. 401].

\section{Список литературы}

1. Сакс С. Теория интеграла. М.: ИЛ, 1949.

2. Ostaszewski K. M. Henstock integration in the plane // Amer. Math. Soc. Memoirs. 1986. V. 63. № 353 .

3. Thomson B. S. Derivates of Interval Functions // Amer. Math. Soc. Memoirs. 1991. V. 93. № 452 .

4. Thomson B.S. Symmetric Properties of Real Functions // Monographs and Textbooks in Pure and Appl. Math. № 183. Marcel Dekker Inc., 1994.

5. Henstock $R$. Theory of Integration. London: Butterworth, 1963.

6. Хинчин A.Я. О процессе интегрирования Данжуа // Матем. сб. 1918. Т. 30. № 4. C. $548-557$.

7. Скляренко В.А. Об интегрируемых по Данжуа суммах всюду сходящихся тригонометрических рядов // ДАН СССР. 1973. Т. 210. №3. С. 533-536.

Поступило в редакцию 25.IX.1995 